Pacific Journal of Mathematics

INTERPOLATION SETS FOR ANALYTIC FUNCTIONS

oo Davit and ARne Stray 


\title{
INTERPOLATION SETS FOR ANALYTIC FUNCTIONS
}

\author{
A. M. Davie ANd A. Stray
}

\begin{abstract}
Let $U$ be a bounded open subset of the complex plane $C$. Criteria are obtained for a subset $E$ of $\bar{U}$ to be an interpolation set for the algebra of all bounded analytic functions on $U$ extending continuously to $E$.
\end{abstract}

In the case where $U$ is the open unit disc $\Delta$, this problem was treated by Détraz [3]. She showed that if $E$ is a subset of the unit circle $T$ then every bounded continuous function on $E$ is the restriction of a bounded analytic function on $A$, extending continuously to $E \cup$ $(T / \bar{E})$, if and only if $E$ has measure zero. We extend this result to any $U$ with connected complement, replacing linear measure on $T$ by harmonic measure (Theorem 1). For the general case the same method yields a criterion in terms of representing measures for $A(U)$ (Theorem 2). Finally in Theorem 3 we use a localization argument to sharpen Theorem 1 and also treat the case where $E$ contains points of $U$ as well as $\partial U$.

Notation. If $S$ is a plane set then $\bar{S}$ denotes its closure and $\partial S$ its boundary. $A(U)$ denotes the algebra of all continuous functions on $\bar{U}$, analytic on $U ; H^{\infty}(U)$ denotes the algebra of all bounded analytic functions on $U ; H_{E}^{\infty}(U)$ denotes the algebra of all bounded continuous functions on $U \cup E$ which are analytic on $U$. If $y \in \bar{U}$, a representing measure for $y$ with respect to $A(U)$ is a positive borel measure $\mu$ on $\bar{U}$ such that $f(y)=\int f d \mu$ for all $f \in A(U)$. We denote by $\|f\|$ the supremum of the function $f$ over its domain of definition. $\Delta(z, \delta)$ denotes the disc with center $z$ and radius $\delta$.

We say that a set $S \subseteq U \cup E$ is an interpolation set for $H_{E}^{\infty}(U)$ if for any bounded continuous $f$ on $S$ we can find $g \in H_{E}^{\infty}(U)$ with $g \mid S=f$. We say $S$ is a peak interpolation set for $H_{E}^{\infty}(U)$ if for any bounded continuous $f$ on $S$, and open set $V \supseteqq S$, and any $\varepsilon>0$, we can find $g \in H_{L}^{\infty}(U)$ with $g \mid S=f,\|g\| \leqq\|f\|$, and $|g|<\varepsilon$ on $U \backslash V$.

Theorem 1. Suppose $\boldsymbol{C} \backslash U$ is connected. Let $F$ be a subset of $\partial U$ with zero harmonic measure for each point of $U$ (with respect to $U$ ).

Then $F$ is a peak interpolation set for $H_{F^{\prime}}^{\infty}(U)$.

The proof follows from the following lemma.

Lemma. With $U$ and $F$ as in the theorem, let $X$ be a compact 
subset of $\bar{U}, W$ a neighborhood of $X$, and $\varepsilon>0$. Then we can find $f \in H_{F}^{\infty}$ with $\|f\| \leqq 2,|1-f|<\varepsilon$ on $F \cap X$, and $|f|<\varepsilon$ on $U \backslash W$.

Proof. We can find a positive harmonic function $\sigma$ on $U$ such that $\sigma(\zeta) \rightarrow \infty$ as $\zeta \rightarrow z, \zeta \in U$, for each $z \in F$. Let $\tau$ be a harmonic conjugate to $\sigma$ on $U$, and let $\theta=\sigma+i \tau$, an analytic function on $U$. Put $h=\theta /(\theta+1)$. Since $\theta$ has positive real part, $h \in H^{\infty}(U)$ with $\|h\| \leqq 1$. Moreover $h(\zeta) \rightarrow 1$ as $\zeta \rightarrow z, \zeta \in U$, for each $z \in F$; hence we can regard $h$ as an element of $H_{F}^{\infty}(U)$, with $h=1$ on $F$.

Now let $\varphi$ be a continuously differentiable function which is 1 on a neighborhood of $X$ and zero outside $W$, with $\|\varphi\|=1$. Then the function

$$
g_{n}(\zeta)=\varphi(\zeta) h^{n}(\zeta)+\frac{1}{\pi} \int_{U} \frac{h^{n}(z)}{z-\zeta} \frac{\partial \varphi}{\partial \bar{z}} d m(z)
$$

is in $H_{F}^{\infty}(U)$. (See [4], p. 210.) Moreover

$$
\left\|g_{n}-\varphi h^{n}\right\| \leqq \frac{1}{\pi}\left\|\frac{\partial \varphi}{\partial \bar{z}}\right\|\left\|h^{n}\right\|_{L 3(U)} \sup _{\zeta}\left\|\frac{1}{z-\zeta}\right\|_{L^{3 \mid 2(U)}} .
$$

The last term is bounded by a constant depending only on $U$, and $\left\|h^{n}\right\|_{L^{3}} \rightarrow 0$ as $n \rightarrow \infty$ since $|h|<1$ in $U$. Choose $n$ so that $\| g_{n}$ $\varphi h^{n} \|<\varepsilon$ and put $f=g_{n}$. Then $f$ satisfies the requirements of the lemma.

Theorem 1 follows from the lemma in exactly the same way as Theorem 1 follows from Lemma 2 in [2]. (For an alternative approach see the proof of Theorem 4.3 of [3]).

We observe that if $A(U)$ is pointwise boundedly dense in $H^{\infty}(U)$ then using Theorem 2.1 of [5] we can modify the function $f$ in the lemma so that it is in $H_{F \cup(\partial U \backslash \bar{F})}^{\infty}(U)$. Then we can prove that $F$ is a peak interpolation set for $H_{F U(\partial u \backslash \bar{F})}^{\infty}(U)$.

In the general situation (where $C \backslash U$ need not be connected) the same method yields the following result. If $y \in U$ we denote by $M_{y}$ the set of all (positive) representing measures for $y$ with respect to $A(U)$ on $\bar{U}$. We assume $U$ is connected.

THEOREM 2. Let $y \in U$ and $F \subseteq \partial U$. Suppose there is a decreasing sequence $\left\{V_{n}\right\}$ of open sets containing $F$, such that $\mu\left(V_{n}\right) \rightarrow 0$ uniformly for $\mu \in M_{y}$.

Then $F$ is a peak interpolation set for $H_{F}^{\infty}(U)$.

Proof. We may suppose $\mu\left(V_{n}\right)<2^{-n}$ for each $\mu \in M_{y}$. For each $n$ let $\left\{g_{n k}\right\}$ be an increasing sequence of nonnegative continuous functions converging to the characteristic function of $V_{n}$. Then $\int g_{n k} d \mu<2^{-n}$ 
for $\mu \in M_{y}$ and so by Theorem II 2.1 of [4], we can find $h_{n k} \in A(U)$ with $\operatorname{Re} h_{n k} \geqq g_{n k}$ on $\bar{U}, \operatorname{Re} h_{n k}(y)<2^{-n}$, and we can also suppose $\operatorname{Im} h_{n k}(y)=0$. Passing to a subsequence we have $h_{n k} \rightarrow h_{n}$ as $k \rightarrow \infty$, pointwise in $U$, where $h_{n}$ is analytic in $U$ with $\operatorname{Re} h_{n} \geqq 1$ on $V_{n} \cap U$ and $\left|h_{n}(y)\right| \leqq 2^{-n}$, Re $h_{n} \geqq 0$ on $U, \operatorname{Im} h_{n}=0$. By Harnack's inequalities the series $\sum_{n=1}^{\infty} h_{n}$ converges pointwise on $U$ to an analytic function $h$ such that $\operatorname{Re} h \geqq 0$ on $U$ and $\operatorname{Re} h(\zeta) \rightarrow \infty$ as $\zeta \rightarrow z, \zeta \in U$, for each $\zeta \in F$.

The rest of the proof follows Theorem 1 .

Again we observe that if $A(U)$ is pointwise boundedly dense in $H^{\infty}(U)$ then the interpolation can be achieved by functions in $H_{F \cup(\partial U \backslash \bar{F})}^{\infty}(U)$. Moreover under the same assumption the converse to Theorem 2 holds, for if $f$ is as in the definition of peak interpolation set, with $V$ chosen so that $y \notin V$, and $g=1$, then we can choose a neighborhood $W$ of $F$ so that $|1-f|<\varepsilon$ on $U \cap W$; by Theorem 5.1 of [1] we can approximate $f$ to within $\varepsilon$ on compact subsets of $W$ by a sequence $\left\{f_{n}\right\}$ in $A(U)$ with $\left\|f_{n}\right\| \leqq 1$, so that $\mu(W)$ is small for all $\mu \in M_{y}$.

The question naturally arises: suppose $\mu(F)=0$ for all $\mu \in M_{y}$. Must there exist open sets $V_{n} \supseteqq F$ such that $\mu\left(V_{n}\right) \rightarrow 0$ uniformly for $\mu \in M_{y}$ ? This is easily verified if $F$ is $\sigma$-compact (in this case the conclusion of Theorem 2 can be deduced from the fact that each compact subset of $F$ is a peak interpolation set for $A(U)$ ). We have no information of the general case.

Lemma 2. Let $F$ be a subset of $\partial U$ such that for each $z \in F$ there exists $\delta>0$ such that $F_{z}=F \cap\{w:|w-z| \leqq \delta / 2\}$ is a peak interpolation set for $H_{F \cap \Delta(z, \delta)}^{\infty}(U \cap \Delta(z, \delta))$, then $F$ is a peak interpolation set for $H_{F}^{\infty}(U)$.

Proof. First we show that $F_{z}$ is a peak interpolation set for $H_{F}^{\infty}(U)$. Let $g$ be a bounded continuous function on $F_{z}$, let $\varepsilon>0$, and let $V$ be an open neighborhood of $F_{z}$. Choose $f \in H_{F \cap \Delta(z, \delta)}^{H}(U \cap$ $\Delta(z, \delta))$ such that $f=g$ on $F_{z},\|f\|=\|g\|$, and $|f|<\varepsilon$ outside $V \cap$ $\{w:|w-z|<3 \delta / 4\}$.

Choose a continuously differentiable function $\varphi$ such that $\varphi=1$ in a neighborhood of $\{w:|w-z| \leqq 3 \delta / 4\}$ and supp $\varphi \subseteq\{w:|w-z|<$ $\delta\}$. Define

$$
f_{1}(w)=f(w) \varphi(w)+\frac{1}{\pi} \int_{U \cap \Delta(z, \delta)} \frac{f(f)}{\zeta-w} \frac{f(\zeta)}{\zeta-w} d m(\zeta)
$$


where $f(w)$ is defined to be zero outside $(F \cup U) \cap \Delta(z, \delta)$. Then $f_{1} \in$ $H_{F}^{\infty}(U)$ and given $t>0$ we can choose $\varepsilon>0$ so that $\left\|f_{1}-f\right\|<t$. Moreover $\left\|f_{1}\right\| \leqq A\|f\|$, where $A$ is an absolute constant. (See [4], p. 210.) Then we have $\left|f_{1}-f\right|<\varepsilon$ on $F_{z}$ and $\left|f_{1}\right|<\varepsilon$ on $U \backslash V$. It now follows by a standard argument (see e.g. [2], Theorem 1), that $F_{z}$ is a peak interpolation set for $H_{F}^{\infty}(U)$.

Now let $V$ be an open set containing $F$. Shrinking $V$ if necessary we may suppose that $V$ is contained in the union of the $\operatorname{discs} \Delta(z, \delta), z \in F$, constructed above. This implies that for any compact set $K \cong V$, we have $K \cap F \leqq \bigcup_{i=1}^{n} F_{z_{i}}$ for some $z_{1}, \cdots, z_{n} \in F$, which easily implies that $K \cap F$ is a peak interpolation set for $H_{F}^{\infty}(U)$. The lemma now follows by the argument used to deduce Theorem 1 from Lemma 3 in [2].

We say that $U$ is locally simple connected at a point $z \in \partial U$ if there exists $\delta>0$ such that $C \backslash(U \cap \Delta(z, \delta))$ is connected. For example, if the diameters of the components of $C \backslash U$ are bounded away from zero then $U$ is locally simply connected at each point of $\partial U$. (Note that $U \cap \Delta(z, \delta)$ is not required to be connected; we only require that each component be simply connected.)

THEOREM 3. Let $S$ be a subset of $\bar{U}$ such that $U$ is locally simply connected at each point of $S \cap \partial U$. Then $S$ is an interpolation set. for $H_{S \cap \partial U}^{\infty}(U)$ if and only if:

(i) $U \cap S$ is an interpolating sequence for $H^{\infty}(U)$,

(ii) $S \cap \partial U$ has zero harmonic measure for each point of $U$, with respect to $U$.

Proof. Assume first that $S$ is an interpolation set for $H_{S(1) U}^{\infty}(U)$. A simple normal family argument shows that (i) holds.

Now let $y \in U$ and choose $f \in H_{S \cap \partial U}^{\infty}(U)$ such that $\|f\| \leqq 1, f=0$ on $S \cap \partial U$, and $f(y) \neq 0$. Then - log $|f|$ is a positive superharmonic function on $U$, tending to $\infty$ at each point of $S \cap \partial U$, and finite at $y$. Thus $S \cap \partial U$ has zero harmonic measure for $y$ with respect to $U$ which proves (ii).

Now assume (i) and (ii) hold, and let $f$ be a bounded continuous function on $S$ with $\|f\| \leqq 1$. By Lemma 2 and Theorem 1, $\partial U \cap S$ is a peak interpolation set for $H_{\partial U \cap S}^{\infty}(U)$ so we can find $h \in H_{\partial U \cap S}^{\infty}(U)$ with $\|h\| \leqq 1$ and $h=f$ on $\partial U \cap S$. Let $g_{1}=f-h$ on $S$, then $g_{1}=0$ on $\partial U \cap S$ so that for any $\varepsilon>0$ we can find $F \in H_{\partial U \cap S}^{\infty}(U)$ so that $F=0$ on $\partial U \cap S,|1-F|<\varepsilon$ on $\left\{z \in S:\left|g_{1}(z)\right|>\varepsilon\right\},\|F\| \leqq 2$. Then $\mid F g_{1}$ $g_{1} \mid \leqq 3 \varepsilon$ on $S$. Choose $G \in H^{\infty}(U)$ so that $\|G\| \leqq M\left\|g_{1}\right\| \leqq 2 M$ and $G=g_{1}$ on $S \cap U$, where $M$ is the interpolation constant of $S \cap U$; then $F G \in H_{\partial U \cap S}^{\infty}$ and satisfies $\left|F G-g_{1}\right| \leqq 3 \varepsilon$ on $S$. Let $\widetilde{f}=F G+h \epsilon$ $H_{\partial U \cap S}^{\infty}(U)$, then $|\widetilde{f}-f| \leqq 3 \varepsilon$ on $S$ and $\|\widetilde{f}\| \leqq 4 M+1$, so the theorem. 
follows by choosing $\varepsilon$ with $3 \varepsilon<1$.

\section{REFERENCES}

1. A. M. Davie, T. W. Gamelin and J. Garnett, Distance estimates and bounded pointwise density, to appear.

2. A. M. Davie and B. K. Øksendal, Peak interpolation sets for some algebras of analytic functions, to appear.

3. J. Détraz, Algèbres de fonctions analytiques dans le disque, Ann. Sci. École Norm. Sup. 3 (1970), 313-352.

4. T. W. Gamelin, Uniform Algebras, Prentice Hall, Englewood Cliffs, N. J., 1969.

5. A. Stray, Approximation and interpolation, Pacific J. Math., to appear.

Received June 7, 1971. The preparation of this paper was supported in part by NSF Grant \#GP-19067.

University of CALifornia, Los ANGeles

AND

UNIVERSITY OF OSLO, OSLO, NORWAY 



\section{PACIFIC JOURNAL OF MATHEMATICS}

\section{EDITORS}

H. SAMELSON

Stanford University

Stanford, California 94305

C. R. HobBY

University of Washington

Seattle, Washington 98105
J. DUGUNDJI

Department of Mathematics

University of Southern California

Los Angeles, California 90007

RICHARD ARENS

University of California

Los Angeles, California 90024

\section{ASSOCIATE EDITORS}

E. F. BECKENBACH

B. H. NeumanN

F WoLF

K. YoshidA

\section{SUPPORTING INSTITUTIONS}

UNIVERSITY OF BRITISH COLUMBIA

UNIVERSITY OF SOUTHERN CALIFORNIA

CALIFORNIA INSTITUTE OF TECHNOLOGY

UNIVERSITY OF CALIFORNIA

MONTANA STATE UNIVERSITY

STANFORD UNIVERSITY

UNIVERSITY OF NEVADA

NEW MEXICO STATE UNIVERSITY

OREGON STATE UNIVERSITY

UNIVERSITY OF OREGON

OSAKA UNIVERSITY

UNIVERSITY OF TOKYO

UNIVERSITY OF UTAH

WASHINGTON STATE UNIVERSITY

UNIVERSITY OF WASHINGTON

$\stackrel{*}{*} \stackrel{*}{*} \stackrel{*}{*}{ }^{*}{ }^{*}$ MMEICAN MATHEMATICAL SOCIETY

NAVAL WEAPONS CENTER

Printed in Japan by International Academic Printing Co., Ltd., Tokyo, Japan 


\section{Pacific Journal of Mathematics}

\section{Vol. 42, No. $1 \quad$ January, 1972}

Tage Bai Andersen, On Banach space valued extensions from split faces ........

David Marion Arnold, A duality for quotient divisible abelian groups of finite

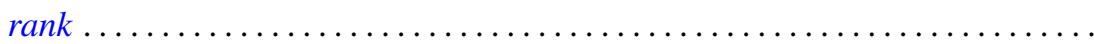

Donald Pollard Ballou, Shock sets for first order nonlinear hyperbolic

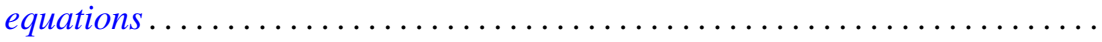

Leon Brown and Lowell J. Hansen, On the range sets of $H^{p}$ functions .........

Alexander Munro Davie and Arne Stray, Interpolation sets for analytic

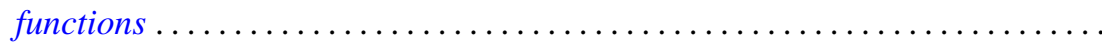

M. G. Deshpande, Structure of right subdirectly irreducible rings. II . . . . . . . . .

Barry J. Gardner, Some closure properties for torsion classes of abelian

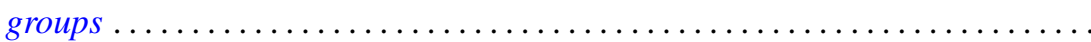

Paul Daniel Hill, Primary groups whose subgroups of smaller cardinality are

direct sums of cyclic groups . . . . . . . . . . . . . . . . . . .

Richard Allan Holzsager, When certain natural maps are equivalences .........

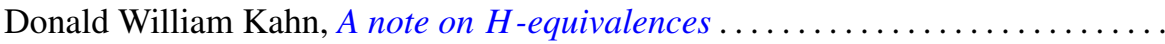

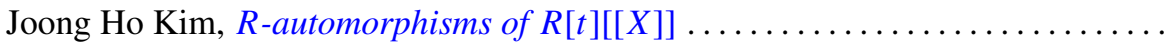

Shin'ichi Kinoshita, On elementary ideals of polyhedra in the 3-sphere.........

Andrew T. Kitchen, Watts cohomology and separability...

Vadim Komkov, A technique for the detection of oscillation of second order

ordinary differential equations .

Charles Philip Lanski and Susan Montgomery, Lie structure of prime rings of characteristic 2

Andrew Lenard, Some remarks on large Toeplitz determinants . .

Kathleen B. Levitz, A characterization of general Z.P.I.-rings. II .

Donald A. Lutz, On the reduction of rank of linear differential systems

David G. Mead, Determinantal ideals, identities, and the Wronskian ...

Arunava Mukherjea, A remark on Tonelli's theorem on integration in product

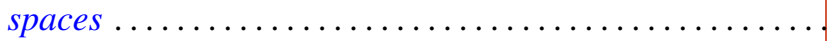

Hyo Chul Myung, A generalization of the prime radical in nonassociative rings.

John Piepenbrink, Rellich densities and an application to unconditionally nonoscillatory elliptic equations.

Michael J. Powers, Lefschetz fixed point theorems for a new class of multi-valued maps .

Aribindi Satyanarayan Rao, On the absolute matrix summability of a Fourier

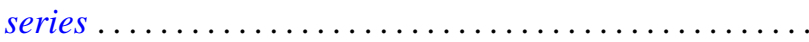

T. S. Ravisankar, On Malcev algebras ......................... 227

William Henry Ruckle, Topologies on sequences spaces . . . . . . . . . . . . . 235

Robert C. Shock, Polynomial rings over finite dimensional rings . . . . . . . . . 251

Richard Tangeman, Strong heredity in radical classes . . . . . . . . . . . . . . 259

B. R. Wenner, Finite-dimensional properties of infinite-dimensional spaces . . . . 267 